\title{
Production of a chaotic squeezed state from a "pion liquid" and overbunching of identical pion correlations
}

\author{
I.V. Andreev ${ }^{1}$ and R.M. Weiner ${ }^{2} \dagger$ \\ ${ }^{1}$ P.N. Lebedev Physical Institute, Moscow, Russia \\ ${ }^{2}$ Physics Department, University of Marburg, Marburg, F.R. Germany
}

\begin{abstract}
It is shown that a one to one correspondence between quantum fields in two different "phases" as might be realized for pions produced from a "hadron liquid" leads to squeezed states. The single and double inclusive cross sections for at chaotic superposition of such states are calculated. The correlation of identical pions is overbunched in comparison with canonical Bose-Einstein correlations.
\end{abstract}

Recent experimental data [1] on identical pion correlations in nucleon-antinucleon annihilation in rest indicate that the intercept of the second order correlation function $C_{2}(p, p)$ reaches values much larger than the "canonical" maximum value of 2 observed in other reactions. This effect which we shall call "overbunching" was interpreted in ref. [2] in terms of resonance production. In this interpretation the space-time information which identical particle interferometry provides through the analogy with the Hanbury BrownTwiss (HBT) effect in optics, i.e. Bose-Einstein correlations (BEC) is lost. While the resonance production is certainly a factor to be considered for annihilation processes, in the present paper an alternative mechanism for the overbunching effect will be proposed in terms of squeezed states, which maintains the link with the HBT effect and which may have other implications in particle physics."] At the same time the derivation of the correlation function for squeezed states given below is a new result also in quantum optics.

We consider pion production via the decay of a stronly interacting pionic system. We suggest that, due to fluctuations, a blob of pionic matter created in particle collision,

\footnotetext{
*E.mail: andreev@lpi.ac.ru

${ }^{\dagger}$ E.mail: weiner@mailer.uni-marburg.de

${ }^{1}$ Note that as shown in 3] resonance production in itself does not necessarily spoil the conventional interpretation of identical particles correlations in terms of BEC. This is the case only for particular models like that considered in [2].
} 
undergoes a sudden breakup into free pions. In other words, the pionic system, having its specific ground state and elementary pionic excitations (not coinciding exactly with the usual vacuum and free particles) converts rapidly into free pions. In this case the single and higher order inclusive cross section and the many-particle correlation functions will depend on the spectrum of excitations in the pionic system. The importance of the form of the spectrum of pionic excitations for multiparticle production was stressed some time ago in ref. [4] where arguments were given for a softening of the spectrum of pions in hot hadronic matter, that is, for a given momentum $\vec{p}$, the effective pion energy in the medium was asserted to be less than the free pion energy.

We shall argue below that the above physical picture results in the production of quantum squeezed states. These states represent a $U(1,1)$ group extension of the coherent states, allowing for incertitudes in momentum (coordinate) below those obtained for coherent states (cf. e.g. [5]). It is known that squeezing may lead to an enhancement of particle correlations, but so far the second order correlation function $C_{2}\left(p_{1}, p_{2}\right)$ is known only for pure squeezed states. The creation of this kind of states was conjectured recently in ref. [6] in the framework of a disoriented chiral pion condensate. Unlike ref. [6], we shall consider the more general case that the pion system may be (partially) chaotic (hot) and thus an averaging over pion states has to be performed. A chaotic distribution is equivalent to a Gaussian form of the density matrix in the $P$ representation (cf. below) which in its turn follows in the assumption that the number of independent sources is large. The coherent and chaotic distributions are the two most interesting extremes in particle physics and quantum optics (cf. [0] where the chaotic distribution of squeezed states, i.e. the "thermal" squeezed states were introduced).

We consider the transition from a pionic "liquid" to a free pion field in the spirit of local parton-hadron duality, i.e. we conjecture a close correspondence between particles (fields) in the two "phases". At the moment of this transition we postulate the following relations between the generalized coordinate $Q$ and the generalized momentum $P$ of the field:

$$
\begin{aligned}
Q & =\frac{1}{\sqrt{2 E_{b}}}\left(b^{+}+b\right)=\frac{1}{\sqrt{2 E_{a}}}\left(a^{+}+a\right) \\
P & =i \sqrt{\frac{E_{b}}{2}}\left(b^{+}-b\right)=i \sqrt{\frac{E_{a}}{2}}\left(a^{+}-a\right)
\end{aligned}
$$

$a^{+}, a$ are the free field creation and annihilation operators and $b^{+}, b$ the corresponding operators in the "liquid". Eq. (11) holds for each mode $p$. Then we get immediately a connection between the $a$ and $b$ operators,

$$
\begin{aligned}
a & =b \cosh r+b^{+} \sinh r, \\
a^{+} & =b \sinh r+b^{+} \cosh r
\end{aligned}
$$


with

$$
r=r(\vec{p})=\frac{1}{2} \log \left(E_{a} / E_{b}\right)
$$

The transformation (2) is just the squeezing transformation [5] with a momentum dependent squeezing parameter $r(\vec{p})$ given by eq. (3) and the coherent eigenstate $\mid \beta>_{b}$ of the $b$-operator is the squeezed state $\mid \alpha, r>_{a}$ of the $a$-operator:

$$
\left|\beta>_{b}=\right| \alpha, r>_{a}
$$

where $\alpha$ and $\beta$ are related by the same transformation (2) as the $a$ and $b$ operators.

In general the system may not be in a pure coherent or squeezed state and then a statistical averaging has to be performed both with respect to the coherent as well as for the squeezed states. In practice it is easier to express the $a, a^{+}$-operators through the $b, b^{+}$-operators according to eq. (2) and then perform the averaging over the coherent states $\mid \beta>_{b}$. Considering charged identical pions (complex valued field) we shall use the Glauber-Sudarshan representation [8] of the density matrix, and write the average value of an operator $\hat{O}$ as

$$
<\hat{O}\left(a, a^{+}\right)>=\prod_{\vec{p}} \int d^{2} \beta_{k} P\{\beta(\vec{p})\}<\beta\left|\hat{O}\left(a\left(b, b^{+}\right), a^{+}\left(b, b^{+}\right)\right)\right| \beta>_{b}
$$

and assume a Gaussian form for the weight function $P\{\beta(\vec{p})\}$. Due to linearity of the squeezing transformation (2) this form will hold also for the $a, a^{+}$-operators.

A blob of decaying pionic matter (particle source) will be characterized by a primordial correlator determined by the number density $n(p)$ and by a function $f(\vec{x})$ describing its geometrical form, see refs. [9, 10]. To make contact with the previous results of [9, 10] we note that the radius of the source $R$ enters the function $f$ and the correlation length $L$ appears in $n(p)$. For simplicity we shall not consider the time dependence here and take the form function $f(\vec{x})$ to be dependent only on the space coordinates.

The direct substitution of the transformation (2) into eq. (5) leads to undefined (divergent) expressions of the form $\delta(0)$ when one tries to perform the normal ordering of $b, b^{+}$ operators (the last is necessary to use the coherent state representation of eq. (5)). To avoid this situation we introduce new creation and annihilation operators which are nonzero only inside the volume of the particle source,

$$
\tilde{a}(\vec{x})=a(\vec{x}) f(\vec{x}) \quad, \quad \tilde{a}^{+}(\vec{x})=a^{+}(\vec{x}) f(\vec{x})
$$

or, for Fourier transformed quantities,

$$
\tilde{a}(\vec{p})=\int \frac{d^{3} k}{(2 \pi)^{3}} a(\vec{k}) f(\vec{k}-\vec{p}) \quad, \quad \tilde{a}^{+}(\vec{p})=\int \frac{d^{3} k}{(2 \pi)^{3}} a^{+}(\vec{k}) f(\vec{p}-\vec{k})
$$


with standard commutation relations

$$
\left[a\left(\vec{p}_{1}\right), a^{+}\left(\vec{p}_{2}\right)\right]=(2 \pi)^{3} \cdot \delta^{3}\left(\vec{p}_{1}-\vec{p}_{2}\right)
$$

Then the equal momentum commutators of the modified operators are finite. For example:

$$
\left[\tilde{a}(\vec{p}), \tilde{a}^{+}(\vec{p})\right]=\int \frac{d^{3} k}{(2 \pi)^{3}} f(\vec{p}-\vec{k}) f(\vec{k}-\vec{p})=\int d^{3} x|f|^{2}(\vec{x})=V_{\text {eff }}
$$

being equal to an effective volume $V_{\text {eff }}$ of the particle source. While this finite size is quite natural in particle physics, it is not so in optics where the system is usually macroscopic.

With the smoothed operators $\tilde{a}(\vec{p}), \tilde{a}^{+}(\vec{p})$ substituted into eq. (5) the form of the source is already taken into account and the remaining statistical averaging may be performed in the same way as for an infinite medium. We have checked that the above procedure reproduces correctly previous results of refs. [9, 10] on correlation functions (at least for the case when the time dependence is omitted).

Now the evaluation of the averaged matrix elements is straightforward. Substituting eqs. (7) and (2) into eq. (5) and performing the Gaussian averaging over coherent states $\mid \beta>_{b}$ we get the single-particle inclusive density in the form:

$$
\begin{aligned}
\rho_{1}(\vec{p}) & =\frac{(2 \pi)^{3}}{\sigma} \frac{d \sigma^{i n}}{d^{3} p}=<\tilde{a}^{+}(\vec{p}) \tilde{a}(\vec{p})> \\
& =\int \frac{d^{3} k}{(2 \pi)^{3}}\left[n_{b}(\vec{k}) \cosh 2 r(\vec{k})+\sinh ^{2} r(\vec{k})\right] f(\vec{p}-\vec{k}) f(\vec{k}-\vec{p})
\end{aligned}
$$

where the function $f$ describes the effect of finite size of the particle source and $n_{b}(\vec{k})$ given by the equation

$$
<\beta^{*}(\vec{k}) \beta\left(\vec{k}^{\prime}\right)>=(2 \pi)^{3} \delta^{3}\left(\vec{k}-\vec{k}^{\prime}\right) n_{b}(\vec{k})
$$

represents the density of pionic "quasiparticles" (b-quanta) (in particular, for a thermal source the function $n_{b}(\vec{k})$ is the usual Planck distribution function).

The squeezed state effect is reflected in eq. (10) in the factor $\cosh 2 r$ multiplying the primary pionic density $n_{b}(\vec{k})$ and in the term $\sinh ^{2} r$ representing the ground state contribution. That is the final state pions are produced even if the pions in the pionic source are absent (zero temperature), just due to the decay of the squeezed vacuum state. According to eq. (10), the single particle density may be strongly enhanced in the presence of squeezed states if the squeezing parameter $r(\vec{p})$ is large enough.

We consider now the two-particle inclusive density

$$
\rho_{2}\left(\vec{p}_{1}, \vec{p}_{2}\right)=\frac{(2 \pi)^{6}}{\sigma} \cdot \frac{d \sigma}{d^{3} p_{1} \cdot d^{3} p_{2}}=<\tilde{a}^{+}\left(\vec{p}_{1}\right) \tilde{a}^{+}\left(\vec{p}_{2}\right) \tilde{a}\left(\vec{p}_{1}\right) \tilde{a}\left(\vec{p}_{2}\right)>
$$


in the presence of squeezed states. With the finite size cut off the two-particle density is calculated in the same way as the single-particle density. Using Gaussian averaging one gets the simple expression

$\rho_{2}\left(\vec{p}_{1}, \vec{p}_{2}\right)=<\tilde{a}^{+}\left(\vec{p}_{1}\right) \tilde{a}\left(\vec{p}_{1}\right)><\tilde{a}^{+}\left(\vec{p}_{2}\right) \tilde{a}\left(\vec{p}_{2}\right)>+\left|<\tilde{a}^{+}\left(\vec{p}_{1}\right) \tilde{a}\left(\vec{p}_{2}\right)>\right|^{2}+\left|<\tilde{a}\left(\vec{p}_{1}\right) \tilde{a}\left(\vec{p}_{2}\right)>\right|^{2}$

with

$$
\begin{aligned}
<\tilde{a}^{+}\left(\vec{p}_{1}\right) \tilde{a}\left(\vec{p}_{2}\right)> & =\int \frac{d^{3} k}{(2 \pi)^{3}}\left[n_{b}(\vec{k}) \cosh 2 r(\vec{k})+\sinh ^{2} r(\vec{k})\right] f\left(\vec{p}_{1}-\vec{k}\right) f\left(\vec{k}-\vec{p}_{2}\right), \\
<\tilde{a}\left(\vec{p}_{1}\right) \tilde{a}\left(\vec{p}_{2}\right)> & =\int \frac{d^{3} k}{(2 \pi)^{3}}\left[n_{b}(\vec{k})+\frac{1}{2}\right] \sinh 2 r(\vec{k}) f\left(\vec{k}_{1}-\vec{p}_{1}\right) f\left(\vec{k}-\vec{p}_{2}\right),
\end{aligned}
$$

The first term in the right hand side of eq. (13) is the product of single-particle densities $\rho_{1}\left(\vec{p}_{1}\right) \rho_{1}\left(\vec{p}_{2}\right)$, the second term is the exchange contribution characteristic for Bose-Einstein correlations modified by the squeezing factor $r$ (for $r=0$ it coincides with the usual BEC). The third term arises only in the presence of squeezed states (it vanishes for $r=0$ ). This last contribution differs from the "surprising" terms in the two-particle correlation function discussed in refs. [10, 11], which are absent in the case of charged identical pions under consideration and which have another dependence on momenta $\vec{p}_{1}, \vec{p}_{2}$, being maximal at $\vec{p}_{1}+\vec{p}_{2}=0$, and not at $\vec{p}_{1}-\vec{p}_{2}=0$ as is the case for all terms in eq. (13).

As one can see from eqs. (10), (13), (14) the second order correlation function

$$
C_{2}\left(\vec{p}_{1}, \vec{p}_{2}\right)=\rho_{2}\left(\vec{p}_{1}, \vec{p}_{2}\right) / \rho_{1}\left(\vec{p}_{1}\right) \rho_{1}\left(\vec{p}_{2}\right),
$$

is enhanced due to the presence of the third term in the right hand side of eq. (13) and in general the value of the ratio $(15)$ is arbitrarily large. In particular, for $n_{b}(\vec{k})=0$ (that is for cold pionic matter when particle production is the result of the squeezed vacuum decay) and for small values of the squeezing parameter $r(\vec{k})$, one may have $C_{2}>>1$ (however, under these conditions the single particle inclusive cross-section (10) is very small). For $r(\vec{k}) \sim 1$ and $\vec{p}_{1} \cong \vec{p}_{2}$ the ratio (15) is close to three.

In conclusion we see that the investigation of Bose-Einstein correlations and in particular of the overbunching effect may shed light on the the role of sqeezing in pion production and on the momentum dependence of the squeezing parameter which in its turn provides information about the spectrum of pions in pionic matter.

\section{Acknowledgements}

This work was supported in part by the Deutsche Forschungsgemeinschaft and the Russian Fund for Fundamental Research, Project No. 93-02-3815. We are indebted to M. Plümer for instructive discussions and for his participation in the early stages of this work. Useful discussions with Y. Sinyukov are also gratefully acknowledged. 


\section{References}

[1] B. Adler et al., CPLEAR Coll., Z.Phys. C63 (1994) 541.

[2] H.Q. Song et al., Z.f.Phys. A342 (1992) 439.

[3] J. Bolz et al., Phys.Rev. C47 (1993) 3860.

[4] E.V. Shuryak, Phys.Rev. D42 (1990) 1764.

[5] D.F. Walls, G.J. Milburn, "Quantum optics", Springer Verlag Berlin, Heidelberg, 1994.

[6] I.J. Kogan, JETP Lett. 59 (1994) 307.

[7] A. Vourdas, Phys.Rev. A34 (1986) 1986; A. Vourdas and R.M. Weiner, Phys.Rev. A36 (1987) 5866; A. Vourdas and R.M. Weiner, Phys.Rev. D38 (1988) 2209.

[8] R.J. Glauber, Phys.Rev. 131 (1963) 2766. E.C.G. Sudarshan, Phys.Rev.Lett. 10 (1963) 277.

[9] I.V. Andreev, R.M. Weiner, Phys.Lett. 235 (1991) 416.

[10] I.V. Andreev, M. Plümer, and R.M. Weiner, Int.Journ.Mod.Phys. A8 (1993) 4577.

[11] I.V. Andreev, M. Plümer, and R.M. Weiner, Phys.Rev.Lett. 67 (1991) 3475. 